\title{
RED DE COMUNICACION HIBRIDA PARA AUTOMATIZACION DISTRIBUIDA BASADA EN LOGO Y SISTEMA SCADA EN EL CONTEXTO DE LA INDUSTRIA 4.0
}

\author{
Antonio José Calderón Godoy, Isaías González Pérez \\ Escuela de Ingenierías Industriales, Universidad de Extremadura, ajcalde@unex.es, igonzp@unex.es
}

\begin{abstract}
Resumen
La implantación real y efectiva de la Industria 4.0 requiere superar varios retos, entre los cuales se encuentran altas inversiones y manejar la interoperabilidad de los múltiples nodos hardware y software interconectados. En este trabajo se aborda el diseño, programación e implementación de una red de comunicación híbrida cable-inalámbrica para integrar un sistema de automatización distribuido basado en autómatas programables LOGO y un Sistema de Supervisión y Control (SCADA) implantado en un Touch Panel de Siemens. Este sistema gestiona satisfactoriamente la heterogeneidad mediante una red Ethernet/IP y empleando equipos industriales de bajo coste así como dispositivos de propósito general.
\end{abstract}

Palabras Clave: automatización, supervisión, integración, comunicación inalámbrica, Ethernet, Industria 4.0.

\section{INTRODUCCIÓN}

Los Sistemas de Supervisión y Control, SCADA por sus siglas en inglés Supervisory Control and Data Acquisition, son ampliamente utilizados en la industria para la monitorización y gestión de procesos automatizados. Se utilizan junto con autómatas programables industriales (Programmable Logic Controllers, PLCs) que adquieren señales de sensores y comandan actuadores para gobernar dichos procesos. Sin embargo, estos sistemas y dispositivos no se limitan al ámbito industrial si no que también se aplican en campos tan diversos como las instalaciones de energías renovables [3, 5, 11], gestión de aguas [6], Smart Grids [4, 9], y automatización de edificios [7], entre otros [1]. Estos elementos contituyen nodos interconectados mediante redes de comunicación que emplean buses de campo industrial y, cada vez más, Ethernet. El intercambio de datos se realiza a través de la red para lograr una operación eficaz y fiable, configurando un sistema de automatización y supervisión distribuido.
En este sentido, conviene destacar la tendencia hacia la implantación de sistemas de creciente complejidad influenciados por nuevos paradigmas tecnológicos como el Internet de las cosas (Internet of Thing, IoT), el Big Data, o los sistema ciber-físicos (CyberPhysical Systems, CPSs). Como consecuencia, a nivel industrial se está desarrollando la considerada Cuarta Revolución Industrial, comúnmente denominada Industria 4.0 [14]. En este escenario, las factorías son entornos inteligentes en que los CPSs monitorizan y orquestan los procesos físicos mediante la creación de una representación digital del mundo físico, y toman decisiones de control y gestión [8].

Para la implantación real y a gran escala de tan ambicioso y prometedor concepto, es necesario superar varios retos entre los cuales destacan la interoperabilidad [12] y las inversiones necesarias [2]. En cuanto a la interoperabilidad, ésta debe ser gestionada debido al problema de integración de sistemas derivado del uso de gran cantidad de elementos, tanto hardware como software, que se deben comunicar de forma eficiente. Éstos generalmente emplean diferentes protocolos de comunicación, topologías, sistemas operativos, sistemas propietarios y de código abierto, de uso general y de uso específico, etc. Además, dentro del entorno de la Industria 4.0, la conectividad inalámbrica está adquiriendo cada vez mayor protagonismo por las ventajas que aporta, como son flexibilidad y movilidad, además de que será empleada por millones de dispositivos en el IoT [16]. En el caso particular de los sistemas de automatización y control, la comunicación inalámbrica aporta beneficios en términos de complejidad de la instalación, menor cableado y costes asociados, capacidad de reconfiguración del sistema mejorada, entre otros [6]. Respecto a los costes asociados, éstos pueden ser muy elevados tanto para infraestructuras de automatización ya existentes como para las de nuevo desarrollo.

Dentro de las actividades del Grupo de investigación GRASP sobre Industria 4.0, se aporta una propuesta de solución a estas cuestiones. Así, en este trabajo se describe la integración en red de relés programables Siemens LOGO!8 230RCE con paneles Human- 
Machine Interface (HMI) Siemens KTP400 Basic PN 400 (más sofisticados que los HMI que suelen ser usados para este tipo de autómatas), y realizar su comunicación a través de un medio inalámbrico mediante el empleo de routers de banda dual TPLINK AC750 Archer C20.

Esta configuración trata de satisfacer por una parte la cuestión de la interoperabilidad al utilizar una red Ethernet/IP para la conexión de los elementos, que es una característica clave para implantar/alcanzar el concepto de Industria 4.0 en la próxima generación de sistemas de control [17]. Por otra parte, el uso de dispositivos de automatización industrial de bajo coste busca reducir los costes asociados a la introducción de las caracterísiticas de la Industria 4.0.

Para mostrar la aplicabilidad real del sistema propuesto, se va a resolver la automatización, control y supervisión del sistema de riego de un campo de golf empleando los elementos antes descritos. Se trata de conseguir, con un coste reducido, la implantación de este prototipo sin necesidad de ejecutar obras de infraestructura. La interconexión inalámbrica de los sistemas de automatización y supervisión es esencial ya que los diferentes dispositivos estarán separados entre sí unas distancias elevadas (en torno a $100 \mathrm{~m}$. entre dos nodos consecutivos), lo que haría al proyecto ser inviable si se pretendiera una comunicación por cable. A esta limitación, habrían de añadirse las dificultades agregadas debido a los movimientos de tierras necesarios para su instalación en el terreno, que harían que el campo quedara inoperativo durante la implantación de este sistema.

El campo de golf seleccionado para implantar este prototipo es Golf Guadiana, situado en la localidad de Badajoz (Badajoz) a 6 kilómetros del núcleo urbano. En la Figura 1 se puede ver la distribución del campo.

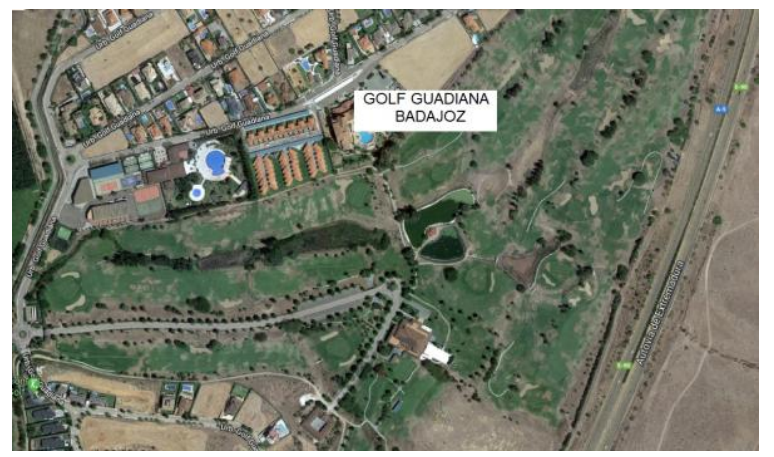

Figura 1: Campo de golf de Badajoz

El sistema de riego utilizará tres bombas para el riego total del campo. La automatización del riego busca también administrar de forma eficiente la potencia de las bombas empleadas para lograr el riego del campo sin necesidad de emplear bombas que sean de una potencia elevada. Para ello, el campo se ha sectorizado en zonas y estas a su vez en áreas, pudiéndose seleccionar de forma independiente las zonas y áreas a regar y tiempo de riego para cada una. El programa de riego desarrollado incluirá tres modos de funcionamiento: Manual, Semiautomático y Automático, que pueden coexistir.

El resto de la contribución se organiza como sigue. La sección 2 describe los elementos hardware y software empleados. En la sección 3 se desarrollan las soluciones propuestas para los sistemas de automatización, supervisión y comunicación. Finalmente, se presentan las conclusiones del trabajo.

\section{HARDWARE/SOFTWARE UTILIZADO}

El sistema de automatización se resuelve utilizando relés programables Siemens LOGO!8 230 RC. El módulo lógico LOGO!8 230 RCE utilizado es el 6ED1052-1FB00-0BA8 de Siemens. Tiene protección IP20 y funciona con alimentación de 115/230 VAC. Se pueden configurar 400 bloques de funciones. Además, dispone de 8 entradas digitales y 4 salidas digitales (relés) y una interfaz Ethernet que puede utilizarse para su programación o para puede comunicarse con otros módulos de la serie LOGO! y dispositivos SIMATIC S7. Cabe mencionar que los autómatas LOGO corresponden a la gama media-baja de Simatic, ofreciendo amplias prestaciones combinadas con un tamaño compacto y un precio reducido.

El sistema de supervisión estará implantado en una pantalla HMI Siemens KTP400 Basic PN, un panel muy económico y de prestaciones suficientes para la aplicación deseada. El panel SIMATIC KTP400 Basic PN - 6AV2123-2DB03-0AX0 tiene una pantalla LCD color de 4" con 65536 colores y se puede manejar gracias a los botones integrados o su pantalla táctil. La comunicación con el PLC se realiza mediante la interfaz Profinet. La configuración del panel KTP400 Basic PN6AV2123-2DB03-0AX0 es posible mediante WinCC Basic V13 o STEP7 BASIC V13.

La comunicación entre los PLCs y el sistema de supervisión y control se va a realizar a través de routers inalámbricos de banda dual TP-LINK AC750 Archer C20i [15]. Éste es un router inalámbrico que implementa el estándar de la próxima generación de Wi-Fi - 802.11ac y compatible con el estándar 802.11n Puede configurarse para funcionar a velocidades de $433 \mathrm{Mbps}$ en la banda de $5 \mathrm{GHz}$ y $300 \mathrm{Mbps}$ en la banda de 2,4 GHz, Estos dispositivos 
proporcionan conectividad a nivel de red o nivel tres en el modelo de referencia Open System Interconection (OSI). Su función principal consiste en enviar o encaminar paquetes de datos de una red a otra, es decir, interconectar subredes, entendiendo por subred un conjunto de máquinas IP que se pueden comunicar sin la intervención de un encaminador (mediante puentes red), y que por tanto tienen prefijos de red distintos. Los routers estarán configurados para trabajar estableciendo una red de conexión punto a punto para conectar los relés programables entre sí y con el sistema de control y supervisión.

En este tipo de red, los dispositivos interconectados actúan como socios iguales o pares entre sí. Como pares, cada dispositivo puede tomar el rol de esclavo o la función de maestro indistitamente.

Todos los dispositivos se conectarán entre sí a través de un switch o conmutador, que es el dispositivo digital lógico de interconexión de equipos que opera en la capa de enlace de datos del modelo OSI. Su función es interconectar dos o más segmentos de red, de manera similar a los puentes de red, pasando datos de un segmento a otro de acuerdo con la dirección MAC de destino de las tramas en la red y eliminando la conexión una vez finalizada. El switch utilizado ha sido el Switch compacto Simatic Net CSM 1277.

La programación y configuración del sistema SCADA han sido resueltas con el paquete software Totally Integrated Automation (TIA) Portal de Siemens, versión V13. El software empleado para configurar y programar los relés programables LOGO ha sido el LOGO!Soft Comfort V8.0.

\section{IMPLEMENTACIÓN}

\subsection{SUBSISTEMA DE AUTOMATIZACIÓN}

Como paso previo a la programación del sistema de automatización, se han de asignar las direcciones IP a cada uno de los dispositivos que forman la subred. Esta tarea se debe resolver de forma manual con los botones de navegación del relé programable LOGO!. La Tabla 1 muestra esta asignación para los tres LOGO! de la zona 1, el panel HMI utilizado y el PC de programación.

Tabla 1: Asignación de IPs

\begin{tabular}{|c|c|}
\hline Aparato & IP \\
\hline Panel HMI & 192.168 .000 .002 \\
\hline LOGO8_A1 & 192.168 .000 .003 \\
\hline LOGO8_A2 & 192.168 .000 .004 \\
\hline LOGO8_A3 & 192.168 .000 .005 \\
\hline PC & 192.168 .000 .245 \\
\hline
\end{tabular}

En la Figura 2: se representa la configuración de la red para los LOGO! de la zona 1. En esta figura también aparecen ya incluidos el PC para programación y configuración y la pantalla donde se ha implantado el sistema SCADA.

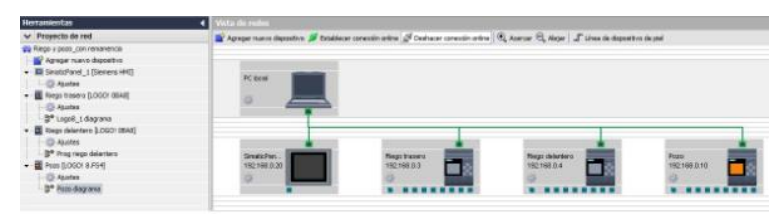

Figura 2: Vista de Red para los LOGO! de la zona 1

Una vez realizada la configuración de la red, se abordará la programación de cada relé programable para el llevar a cabo la automatización del riego de la zona.

Cada LOGO! controlará la activación de la bomba utilizada para la zona determinada, una electroválvula general para la zona y una electroválvula por área. En la Figura 3 aparece la conexión de estos dispositivos de campo con el relé programable.

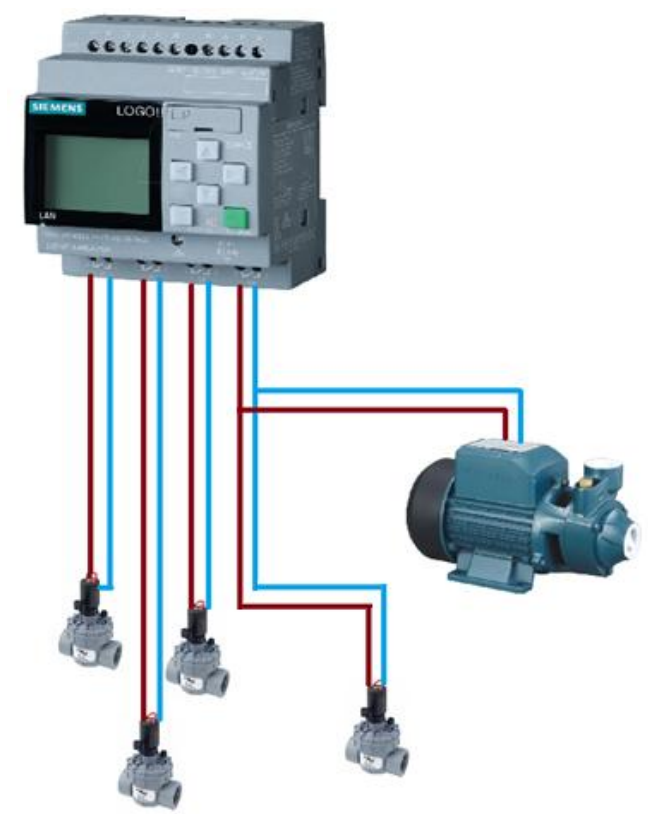

Figura 3: Conexiones de las salidas de un LOGO! con las electroválvulas y la bomba

La estructura en LOGO!Soft es la que aparece en la Figura 4 corresponde a una de las secuencias de riego programadas para los modos semiautomático o automático. 


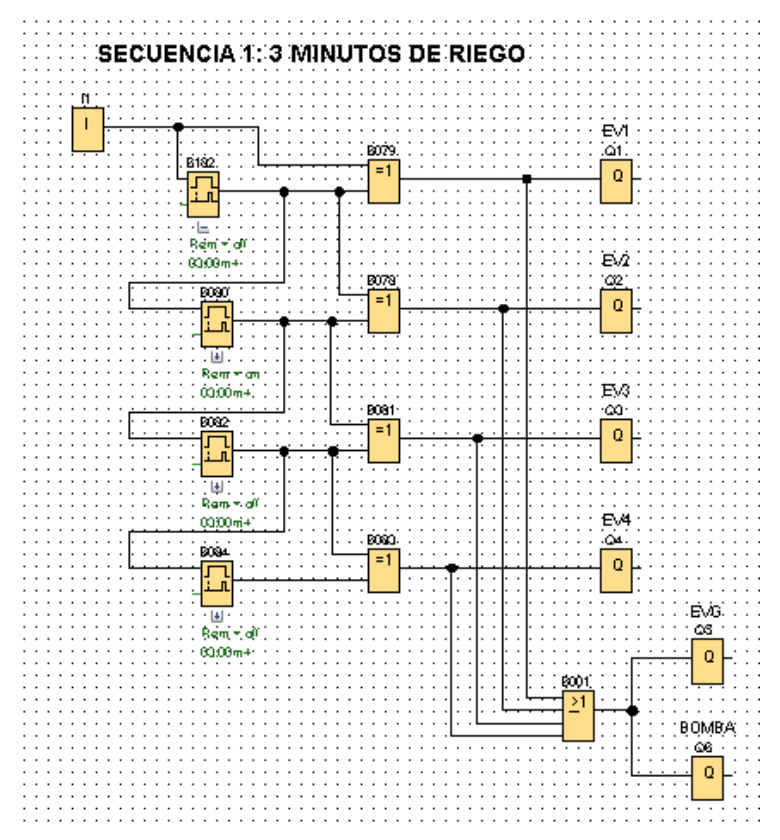

Figura 4: Bloque secuencia de riego de una zona

\subsection{SISTEMA DE SUPERVISIÓN}

En este apartado se aborda la descripción de la configuración y programación del panel HMI Siemens KTP400 Basic PN utilizado para implementar el sistema de supervisión del circuito de riego.

Como se señaló en el apartado precedente para los dispositivos del sistema de automatización, en primer lugar se ha de asignar la dirección IP al panel HMI. En este proyecto se ha asignado a la KTP la IP 192.168.000.002. Para ello, se utilizará el paquete software de Siemens TIA Portal V13.

En este caso, además también se ha de utilizar el software LOGO!Soft Comfort V8.0 para agregar este dispositivo como panel Simatic y asignar la IP que tendrá en la red de automatización del sistema de riego. Esta asignación se muestra en la pantalla de selección que aparece en la Figura 5. El flujo de información entre de ambos sistemas, automatización y supervisión, se resuelve utilizando variables de red en la programación de los relés programables. Estas variables de red habrán de ser definidas también en el panel HMI.

La Figura 6 plasma la configuración de la conexión entre el panel de operador (HMI) y uno de los LOGO!. Posteriormente, se habrán de definir las variables que permitan la interconectividad entre todos los dispositivos que integren la subred de cada área utilizando para ello las conexiones establecidas.

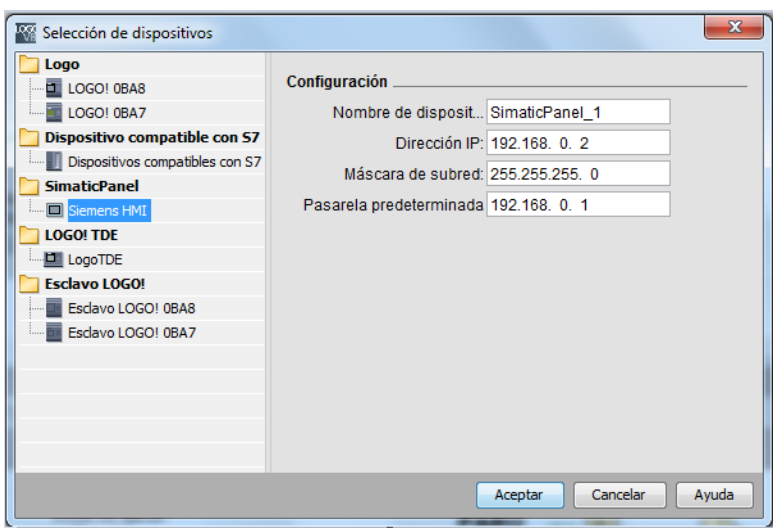

Figura 5: Introducción del panel KTP en la red en LOGO!Soft Comfort V8.0

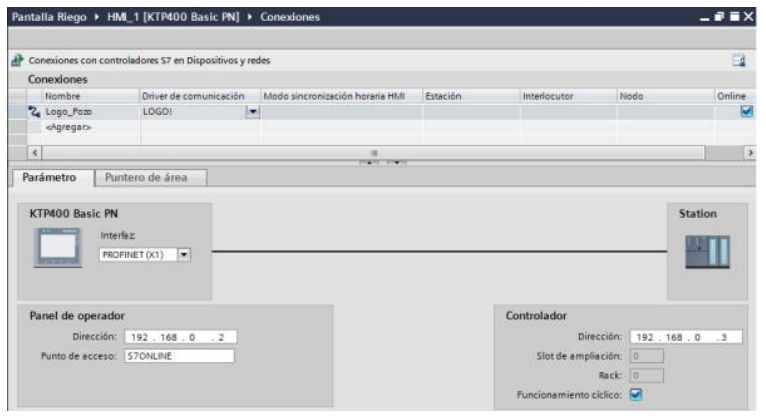

Figura 6: Configuración de la conexión del panel KTP con uno de los LOGO! de la subred

A continuación se confeccionarán las pantallas que conforman la aplicación SCADA desarrollada. En la Figura 7 se muestra la pantalla creada para la selección de una zona de riego cuando se está en el modo manual.

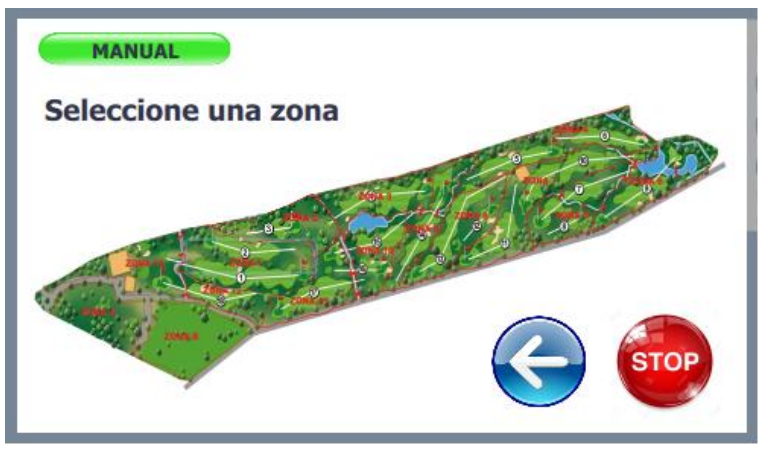

Figura 7: Selección de zona del modo manual

\subsection{CONFIGURACIÓN DEL SUBSISTEMA DE COMUNICACIÓN INALÁMBRICA}

En este apartado se propone una solución para la conexión entre la pantalla de visualización y un relé programable. Para ello, se ha creado un "túnel" wifi a través de dos routers inalámbricos de Banda Dual TP-LINK AC750 Archer, los cuales han sido 
configurados en modo red punto a punto. La configuración de los routers se ha realizado conectando individualmente cada router al PC a través del puerto Ethernet utilizando el asistente de configuración que proporciona el fabricante de los mismos según se aprecia en la Figura 8.

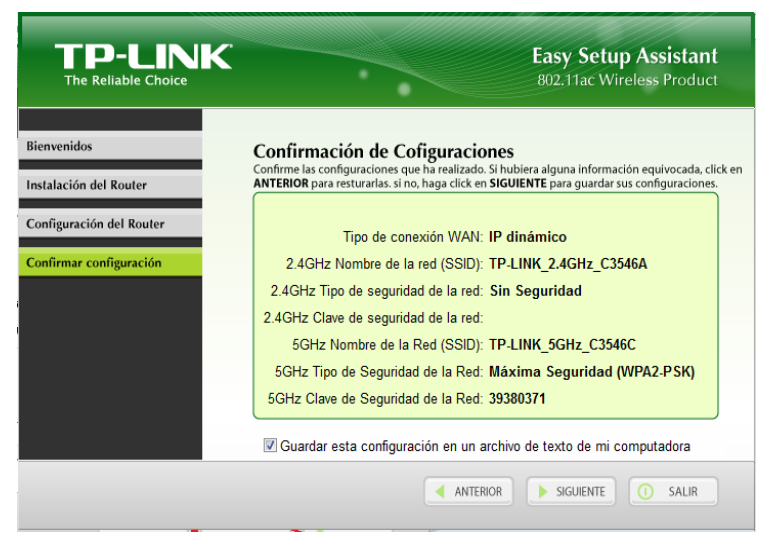

Figura 8: Configuración de un router

Tras concluir la configuración se accede al router a través de su IP para su ajuste y parametrización. Con las opciones de ajuste se selecciona la velocidad de comunicación wireless, el canal de comunicación y se habilita la opción WDS (Wireless Distribution System).

Este sistema posibilita la interconexión inalámbrica de puntos de acceso en una red IEEE 802.11. Permite que ésta pueda ser ampliada mediante múltiples puntos de acceso sin la necesidad de un cable troncal que los conecte. La Figura 9 muestra la pantalla de ajustes del router con las opciones seleccionadas.

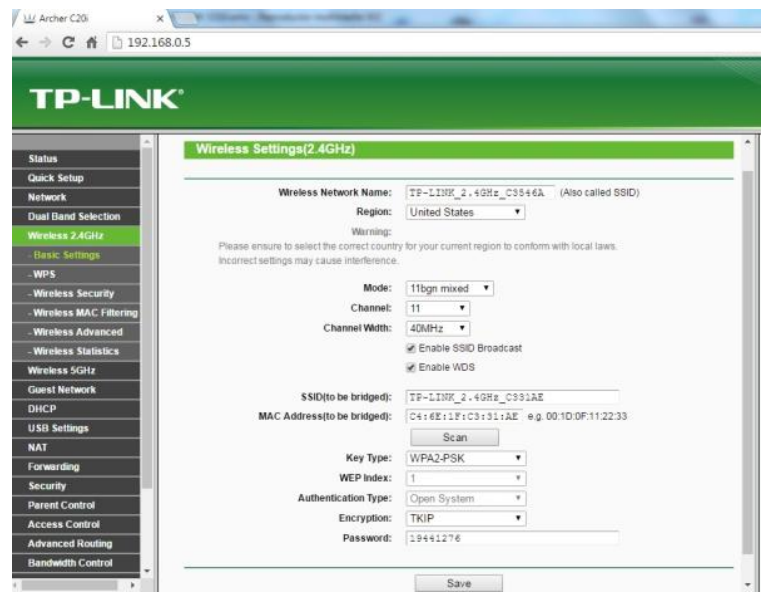

Figura 9: Configuración de un router

En el modelo OSI la información que se envía a través de los routers desde el LOGO! hasta la KTP realiza el "camino" que se señala en la Figura 10.

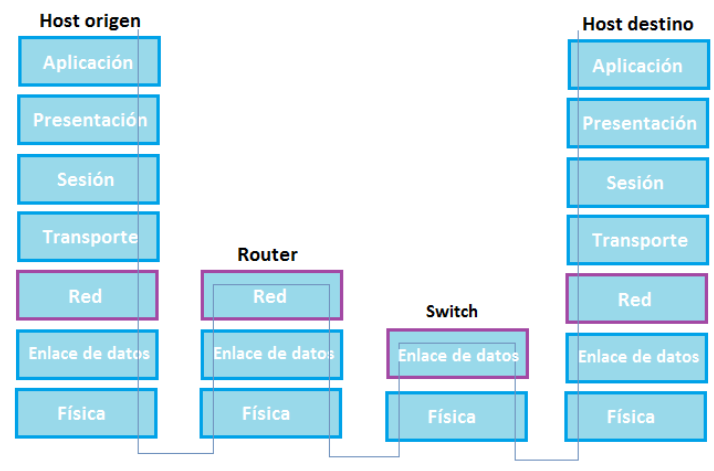

Figura 10: Datos a través de router y switch desde LOGO! hasta KTP

Se ha desarrollado un prototipo, a escala de laboratorio, para probar el funcionamiento del sistema integrado. De esta manera, se asegura el funcionamiento de la red de comunicación híbrida cable/inalámbrica entre los relés programables (LOGO!8) y entre éstos y el sistema de supervisión (SCADA).

La interconexión entre los diferentes dispositivos empleados para llevar a cabo la implementación del prototipo de laboratorio del sistema de automatización y control del riego mediante el uso de relés programables LOGO! se puede apreciar en la Figura 11.

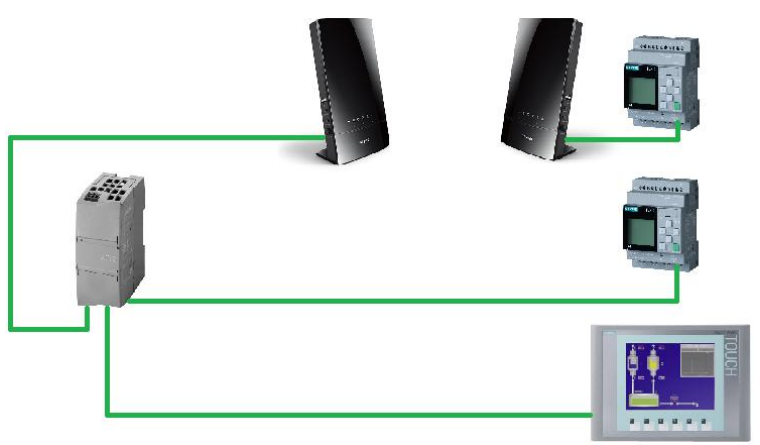

Figura 11: Esquema de conexionado de la red híbrida

En la Figura 12 aparece el prototipo construido a nivel de laboratorio integrando los componentes descritos.

\section{CONCLUSIÓN}

En este trabajo se ha resuelto de manera exitosa la integración de un sistema de automatización basado en relés programables LOGO! de bajo coste y un sistema de supervisión realizado mediante un panel de operador táctil. Además se ha implementado un sistema de comunicación híbrido que combina una conexión cableada con una conexión inalámbrica 
entre los diversos dispositivos empleados en este proyecto mediante la utilización de routers inalámbricos y un switch. Este desarrollo se contextualiza en el escenario de la Industria 4.0 ya que se ha aportado una solución de bajo coste para integrar comunicación inalámbrica con dispositivos de automatización y también de propósito general en una red Ethernet/IP.

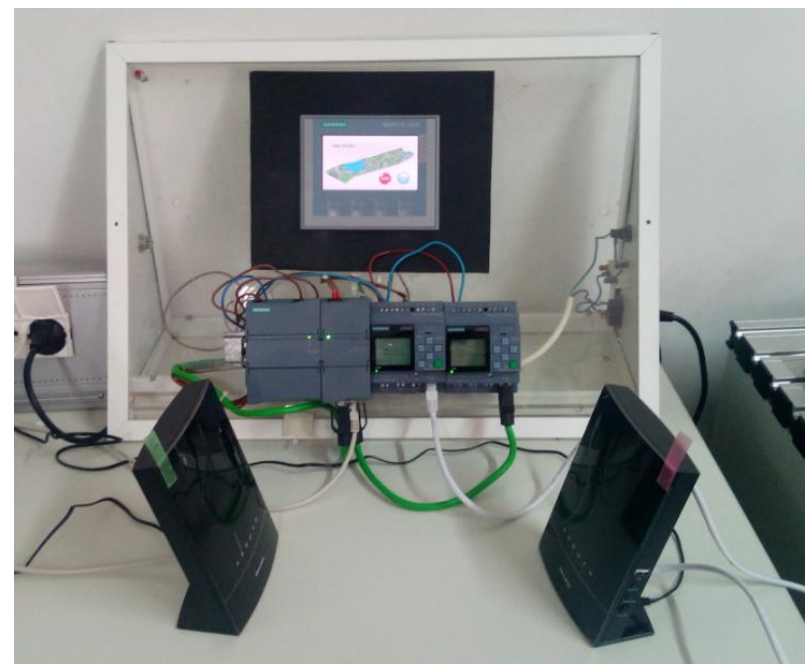

Figura 12: Prueba de comunicación en laboratorio

\section{Agradecimientos}

Este trabajo ha sido financiado con Fondos FEDER (Programa Operativo FEDER de Extremadura 20142020). Ayuda a Grupos de Investigación de la Junta de Extremadura (ref. GR18159).

\section{English summary}

\section{HYBRID COMMUNICATION NETWORK FOR DISTRIBUTED AUTOMATION BASED ON LOGO AND SCADA SYSTEM IN THE INDUSTRY 4.0 CONTEXT}

\section{Abstract}

This paper deals with the design, programming and implementation of a hybrid cable-wireless communication system to integrate a distributed automation system based on LOGO programmable controllers and a Supervisory Control and Data Acquisition (SCADA) system implemented in a Siemens Touch Panel.

Keywords: automation, supervision, integration, wireless connection, Ethernet, Industry 4.0.

\section{Referencias}

[1] Alphonsus, E.R., Abdullah, M.O., (2016) “A review on the applications of programmable logic controllers (PLCs)", Renewable and Sustainable Energy Reviews, vol. 60, pp. 11851205. DOI:10.1016/j.rser.2016.01.025.

[2] Calderón, A.J., González, I., (2018) "Integration of Sensor and Actuator Networks and the SCADA System to Promote the Migration of the Legacy Flexible Manufacturing System towards the Industry 4.0 Concept", Journal of Sensor and Actuator Networks, vol. 7(2), 23. DOI: $10.3390 /$ jsan7020023.

[3] Calderón, M., Calderón, A.J., Ramiro, A., González, J.F., González, I., (2011) "Evaluation of a hybrid photovoltaic-wind system with hydrogen storage performance using exergy analysis", International Journal of Hydrogen Energy, vol. 36, pp. 5751-5762. DOI: 10.1016/j.ijhydene.2011.02.055.

[4] Cintuglu, M.H., Mohammed, O.A., Akkaya, K., Uluagac, A.S., (2017) "A Survey on Smart Grid Cyber-Physical System Testbeds", IEEE Communications Surveys and Tutorials, vol. 19, pp. 446-464. DOI:10.1109/COMST.2016.2627399.

[5] Dispenza, G., Sergi, F., Napoli, G., Randazzo, N., Di Novo, S., Micari, S., Antonucci, V., Andaloro, L., (2017) "Development of a solar powered hydrogen fueling station in smart cities applications", International Journal of Hydrogen Energy, vol. 42(46), pp. 2788427893. DOI: 10.1016/j.ijhydene.2017.07.047.

[6] Ferracuti, F., Freddi, A., Monteriù, A., Prist, M., (2016) "An Integrated Simulation Module for Cyber-Physical Automation Systems", Sensors, vol. 16, pp. 645. DOI: 10.3390/s16050645.

[6] Figueiredo, J., Ayala, M., Rijo, M., (2013) "SCADA system with predictive controller applied to irrigation canals", Control Engineering Practice, vol. 21, 870-886, DOI:10.1016/j.conengprac.2013.01.008.

[7] Figueiredo, J., Sá da Costa, J., (2012) "A SCADA system for energy management in intelligent buildings", Energy and Buildings, vol. 49, 85-98. DOI:10.1016/j.enbuild.2012.01.041.

[8] Givehchi, O., Landsdorf, K., Simoens, P., Colombo, A.W., (2017) "Interoperability for 
industrial cyber-physical systems: an approach for legacy systems", IEEE Transactions on Industrial Informatics, vol. 13(6), pp. 33703378. DOI: 10.1109/TII.2017.2740434.

[9] González, I., Calderón, A.J., Andújar, J.M., (2017) "Novel Remote Monitoring Platform for RES-Hydrogen based Smart Microgrid", Energy Conversion and Management, vol. 148, pp. 489-505.

DOI: 10.1016/j.enconman.2017.06.031.

[10] González, I., Calderón, A.J., Barragán, A.J., Andújar, J.M., (2017) "Integration of Sensors, Controllers and Instruments Using a Novel OPC Architecture", Sensors, vol. 17(7), 1512. DOI: 10.3390/s17071512.

[11] González, I., Calderón, A.J., Calderón Godoy, M., Herrero, J.L., (2015) "Monitoring of Electric Power Systems: Application to selfsufficient Hybrid Renewable Energy Systems", Proceedings of the IEEE 9th International Conference on Compatibility and Power Electronics (CPE), Caparica, Portugal. DOI: 10.1109/CPE.2015.7231137.

[12] González, I., Calderón, A.J., Figueiredo, J., Sousa, J.M.C., (2018) "Design of an educational platform for automation and supervision under the Industry 4.0 framework", Proceedings of the 12th International Technology, Education and Development Conference (INTED), Valencia, Spain.

[13] González, I., Calderón, A.J., Mejías, A., Andújar, J.M., (2016) "Novel networked remote laboratory architecture for open connectivity based on PLC-OPC-LabVIEW-EJS integration. Application to remote fuzzy control and sensors data acquisition", Sensors, vol. 16(11), pp. 1822. DOI: $10.3390 / \mathrm{s} 16111822$.

[14] Hoffmann, E., Rüsch, M., (2017) "Industry 4.0 and the current status as well as future prospects on logistics", Computers in Industry, vol. 89, pp. 23-34.

DOI: 10.1016/j.compind.2017.04.002.

[15] http://www.tp-link.es/products/details/cat9_Archer-C20i.html\#overview

[16] Marin, L., Pawlowski, M.P., Jara, A., (2015) "Optimized ECC Implementation for Secure Communication between Heterogeneous IoT Devices", Sensors, vol. 15, pp. 21478-21499. DOI: $10.3390 / \mathrm{s} 150921478$.
[17] Rogoll, G., Suzuki, T., (2017) "Advance physical layer for digital communication in process industries", Proceedings of 56th Annual Conference of the Society of Instrument and Control Engineers of Japan (SICE), Kanazawa, Japan. DOI: 10.23919/SICE.2017.8105720.

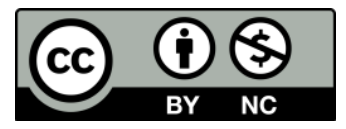

(C) 2018 by the authors. Submitted for possible open access publication under the terms and conditions of the Creative Commons Attribution CC-BY-NC 3.0 license (https://creativecommons.org/licenses/by-nc/3.0). 